\title{
Implicit Multifunction Theorems in Banach Spaces
}

\author{
Ming-ge Yang ${ }^{1,2}$ and Yi-fan $X u^{1}$ \\ ${ }^{1}$ School of Management, Fudan University, Shanghai 200433, China \\ ${ }^{2}$ Department of Mathematics, Luoyang Normal University, Luoyang, Henan 471022, China
}

Correspondence should be addressed to Ming-ge Yang; yang_mingge@163.com

Received 31 December 2013; Accepted 21 January 2014; Published 9 March 2014

Academic Editor: Nan-Jing Huang

Copyright (C) 2014 M.-g. Yang and Y.-f. Xu. This is an open access article distributed under the Creative Commons Attribution License, which permits unrestricted use, distribution, and reproduction in any medium, provided the original work is properly cited.

\begin{abstract}
This paper is mainly devoted to the study of implicit multifunction theorems in terms of Clarke coderivative in general Banach spaces. We present new sufficient conditions for the local metric regularity, metric regularity, Lipschitz-like property, nonemptiness, and lower semicontinuity of implicit multifunctions in general Banach spaces. The basic tools of our analysis involve the Ekeland variational principle, the Clarke subdifferential, and the Clarke coderivative.
\end{abstract}

\section{Introduction}

Let $X$ and $P$ be topological spaces, $Y$ a topological vector space, $F: X \times P \rightrightarrows Y$ a multifunction, and $\left(x_{0}, p_{0}\right) \in X \times P$ a pair with $0 \in F\left(x_{0}, p_{0}\right)$. The multifunction $G: P \rightrightarrows X$ defined by

$$
G(p):=\{x \in X \mid 0 \in F(x, p)\}
$$

is called the implicit multifunction defined by the inclusion $0 \in F(x, p)$. The problem is to find some verifiable conditions on $F$ such that $G$ has the desirable properties. In the literature, different topological, metric, and differential properties (e.g., lower semicontinuity, metric regularity, Lipschitz-like property, upper Lipschitz continuity, and $B$-differentiability) of implicit multifunctions are considered. The structure of $F$ and its behavior around $\left(x_{0}, p_{0}\right)$ decide local properties of $G$ in a neighborhood of the point $\left(p_{0}, x_{0}\right)$ in its graph.

The study of the stability of implicit multifunctions has a long history. The pioneering works of Robinson [1-4] gave good samples for implicit multifunction theorems and their applications. Later, Ledyaev and Zhu [5] and Ngai and Théra [6] established sufficient conditions for the metric regularity of implicit multifunctions in terms of Fréchet coderivative in Banach spaces with Fréchet-smooth Lipschitz bump functions. Recently, Lee et al. [7] showed some sufficient conditions for the nonemptiness, the lower semicontinuity, the metric regularity, and the Lipschitz-like property of implicit multifunctions in terms of Mordukhovich normal coderivative in Asplund spaces. Yen and Yao [8] obtained some point-based sufficient conditions for the metric regularity of implicit multifunctions in finite-dimensional spaces. Huy and Yao [9] established another set of sufficient conditions for the local metric regularity and the Lipschitz-like property of implicit multifunctions in terms of Mordukhovich normal coderivative in Asplund spaces. Huy and Yao [10] studied the metric regularity of implicit multifunctions in terms of Mordukhovich normal coderivative in WCG Asplund spaces. Chieu et al. [11] examined the relationship between the metric regularity and the Lipschitz-like property of implicit multifunctions in finite-dimensional spaces. Chuong [12] gave new sufficient conditions for the Lipschitz-like property of implicit multifunctions in terms of Fréchet coderivative in Asplund spaces. Nghia [13] is also devoted to the study of implicit multifunction theorems in terms of Fréchet coderivative in Asplund spaces. Yang and Huang [14] gave sufficient conditions for the local metric regularity, the metric regularity, the Lipschitz-like property, the nonemptiness, and the lower semicontinuity of random implicit multifunctions in terms of Mordukhovich normal coderivative in separable Asplund spaces.

As mentioned above, the results obtained for the (local) metric regularity, the Lipschitz-like property, the nonemptiness, and the lower semicontinuity of implicit multifunctions are almost restricted in Asplund spaces. Noting that 
Huy et al. [15] established new sufficient conditions for both the metric regularity and the Lipschitz-like property of implicit multifunctions in terms of Clarke coderivative in general Banach spaces, it is worth mentioning that the coderivative condition of implicit multifunction theorems in [15] can be weakened. So it is natural for us to study implicit multifunction theorems under much weaker conditions in terms of Clarke coderivative in general Banach spaces.

In this paper, we present new sufficient conditions for the local metric regularity, metric regularity, Lipschitz-like property, nonemptiness, and lower semicontinuity of implicit multifunctions in general Banach spaces. The basic tools of our analysis involve the Ekeland variational principle, the Clarke subdifferential, and the Clarke coderivative.

The paper is organized as follows. Section 2 recalls some basic concepts and results from variational analysis and generalized differentiation. Section 3 presents some implicit multifunction theorems in terms of Clarke coderivative in general Banach spaces.

\section{Preliminaries}

Throughout this paper, unless otherwise stated, all spaces under consideration are Banach spaces whose norms are always denoted by $\|\cdot\|$. For any $X$, we consider its dual space $X^{*}$ equipped with the weak ${ }^{*}$ topology $w^{*}$, where $\langle\cdot, \cdot\rangle$ means the canonical pairing. As usual, $B_{X}$ and $B_{X^{*}}$ stand for the closed unit balls of the Banach space $X$ and its dual $X^{*}$, respectively. The closed ball with center $x$ and radius $r$ is denoted by $B(x, r)$. For a subset $\Omega \subset X$, int $\Omega$ denote the interior of $\Omega$.

For a closed subset $A$ of $X$ and a point $a \in A$, let $T_{c}(a ; A)$ denote the Clarke tangent cone of $A$ at $a$; that is, $v \in T_{c}(a ; A)$ if and only if, for each sequence $\left\{a_{n}\right\}$ in $A$ converging to $a$ and each sequence $\left\{t_{n}\right\}$ in $(0,+\infty)$ decreasing to 0 , there exists a sequence $\left\{v_{n}\right\}$ in $X$ converging to $v$ such that $a_{n}+t_{n} v_{n} \in A$ for all $n$. We denote by $N_{c}(a ; A)$ the Clarke normal cone of $A$ at $a$; that is,

$$
N_{c}(a ; A):=\left\{x^{*} \in X^{*} \mid\left\langle x^{*}, h\right\rangle \leq 0, \forall h \in T_{c}(a ; A)\right\} .
$$

Let $F: X \rightrightarrows Y$ be a multifunction between topological spaces. Denote by

$$
\begin{gathered}
\operatorname{dom} F:=\{x \in X \mid F(x) \neq \emptyset\}, \\
\operatorname{rge} F:=\{y \in Y \mid \exists x \text { with } y \in F(x)\}
\end{gathered}
$$

the domain and the range of $F$. Each multifunction $F: X \rightrightarrows$ $Y$ is uniquely associated with its graph:

$$
\operatorname{gph} F:=\{(x, y) \in X \times Y \mid y \in F(x)\}
$$

in the product space $X \times Y$. As usual, $F$ is said to be closed if $\mathrm{gph} F$ is a closed subset of $X \times Y$. F is lower semicontinuous (in short, 1.s.c.) at $\bar{x} \in \operatorname{dom} F$ if, for any open set $V \subset Y$ satisfying $F(\bar{x}) \cap V \neq \emptyset$, there exists a neighborhood $U$ of $\bar{x}$ such that
$F(x) \cap V \neq \emptyset$ for all $x \in U$. For any $(x, y) \in \operatorname{gph} F$, the Clarke coderivative $D_{c}^{*} F(x, y)$ of $F$ at $(x, y)$ is defined by

$$
\begin{array}{r}
D_{c}^{*} F(x, y)\left(y^{*}\right):=\left\{x^{*} \in X^{*} \mid\left(x^{*},-y^{*}\right) \in N_{c}((x, y)\right. \\
; \operatorname{gph} F)\}, \\
\forall y^{*} \in Y^{*} .
\end{array}
$$

The history of the coderivatives can be found in $[16,17]$.

Let $\varphi: X \rightarrow \overline{\mathbb{R}}$ be an extended real-valued function,

$$
\begin{gathered}
\operatorname{dom} \varphi:=\{x \in X \mid \varphi(x)<\infty\} \\
\text { epi } \varphi:=\{(x, \mu) \in X \times \mathbb{R} \mid \mu \geq \varphi(x)\} .
\end{gathered}
$$

We say that $\varphi$ is proper if $\varphi(x)>-\infty$ for all $x \in X$ and $\operatorname{dom} \varphi \neq \emptyset$. Recall that $\varphi$ is 1.s.c. at a point $\bar{x}$ with $|\varphi(\bar{x})|<\infty$ if $\liminf _{x \rightarrow \bar{x}} \varphi(x) \geq \varphi(\bar{x})$. We say that $\varphi$ is l.s.c. around $\bar{x}$ when it is l.s.c. at any point of some neighborhood of $\bar{x}$. For $x \in \operatorname{dom} \varphi$ and $h \in X$, let $\varphi^{\uparrow}(x, h)$ denote the generalized directional derivative introduced by Rockafellar (cf. [18]); that is,

$$
\varphi^{\uparrow}(x, h):=\lim _{\varepsilon \downarrow 0} \lim _{\substack{\varphi \\ z \rightarrow x}} \sup _{t \downarrow 0} \inf _{w \in h+\varepsilon B_{X}} \frac{\varphi(z+t w)-\varphi(z)}{t},
$$

where the expression $z \stackrel{\varphi}{\rightarrow} x$ means that $z \rightarrow x$ and $\varphi(z) \rightarrow \varphi(x)$. Let $\partial_{c} \varphi(x)$ denote the Clarke-Rockafellar subdifferential of $\varphi$ at $x$; that is,

$$
\partial_{c} \varphi(x):=\left\{x^{*} \in X^{*} \mid\left\langle x^{*}, h\right\rangle \leq \varphi^{\uparrow}(x, h), \forall h \in X\right\} .
$$

When $\varphi$ is convex, the Clarke-Rockafellar subdifferential reduces to the one in the sense of convex analysis; that is,

$$
\begin{array}{r}
\partial_{c} \varphi(x) \\
=\left\{x^{*} \in X^{*} \mid\left\langle x^{*}, y-x\right\rangle \leq \varphi(y)-\varphi(x), \forall y \in X\right\}, \\
\forall x \in \operatorname{dom} \varphi
\end{array}
$$

For a closed subset $A$ in $X$, let $\delta_{A}$ denote the indicator function of $A$. It is known that $\partial_{c} \delta_{A}(a)=N_{c}(a ; A)$ and

$$
\begin{array}{r}
\partial_{c} \varphi(x)=\left\{x^{*} \in X^{*} \mid\left(x^{*},-1\right) \in N_{c}((x, \varphi(x)) ; \operatorname{epi} \varphi)\right\}, \\
\forall x \in \operatorname{dom} \varphi .
\end{array}
$$

The following sum rule plays important role in variational analysis and is useful for our analysis.

Lemma 1 (see [18]). Let $X$ be a Banach space and let $\varphi_{1}, \varphi_{2}$ : $X \rightarrow \overline{\mathbb{R}}$ be proper lower semicontinuous functions. Let $x \in$ $\operatorname{dom} \varphi_{1} \cap \operatorname{dom} \varphi_{2}$ be a local minimizer of $\varphi_{1}+\varphi_{2}$. Suppose that one of $\varphi_{1}$ and $\varphi_{2}$ is locally Lipschitz around $x$. Then

$$
0 \in \partial_{c} \varphi_{1}(x)+\partial_{c} \varphi_{2}(x)
$$


Lemma 2 (see [16]). Let $(X, d)$ be a metric space. Assume that $X$ is complete and that $\varphi: X \rightarrow \mathbb{\mathbb { R }}$ is a proper l.s.c. function bounded from below. Let $\varepsilon>0$ and $x_{0} \in X$ be given such that $\varphi\left(x_{0}\right) \leq \inf _{X} \varphi+\varepsilon$. Then for any $\lambda>0$ there is $\bar{x} \in X$ satisfying
(a) $\varphi(\bar{x}) \leq \varphi\left(x_{0}\right)$
(b) $d\left(\bar{x}, x_{0}\right) \leq \lambda$,
(c) $\varphi(x)+(\varepsilon / \lambda) d(x, \bar{x})>\varphi(\bar{x})$ for all $x \neq \bar{x}$.

\section{Implicit Multifunction Theorems}

Theorem 3. Let $X$ and $Y$ be Banach spaces, $P$ a topological space, $F: X \times P \rightrightarrows Y$ a multifunction, $G: P \rightrightarrows X$ the implicit multifunction defined by (1), and $\left(x_{0}, p_{0}\right) \in X \times P$ a pair with $0 \in F\left(x_{0}, p_{0}\right)$. Denote $F_{p}(\cdot):=F(\cdot, p)$. Suppose that there exist constants $r>0$ and $\sigma>0$ such that

(i) for any $p \in B\left(p_{0}, r\right)$, the multifunction $F_{p}(\cdot)$ is closed;

(ii) for any $\delta>0$ and any $(x, p) \in B\left(x_{0}, r\right) \times B\left(p_{0}, r\right)$ with $0 \notin F(x, p)$,

$$
\begin{aligned}
\sigma \leq \lim _{\delta \downarrow 0} \inf \left\{\left\|x^{*}\right\|: x^{*} \in D_{c}^{*} F_{p}(x, y)\left(y^{*}\right),\right. \\
\left.y \in \Pi_{\delta}\left(0 ; F_{p}(x)\right) \cap B(0, r), y^{*} \in J_{\delta}(y)\right\},
\end{aligned}
$$

where $\Pi_{\delta}\left(0 ; F_{p}(x)\right):=\left\{\begin{array}{llll}y & \in F_{p}(x) \quad \mid\|y\| \leq\end{array}\right.$ $\left.\operatorname{dist}\left(0, F_{p}(x)\right)+\delta\right\}$ and $J_{\delta}(y):=\left\{y^{*} \in S_{Y^{*}} \mid\|y\|-\right.$ $\left.\left\langle y^{*}, y\right\rangle \leq \delta\right\}$.

Then $G$ is locally metrically regular around $\left(x_{0}, p_{0}\right)$ with modulus $1 / \sigma$. In fact, for any $\mu \in(0, \min \{r, r \sigma / 2\})$, we have

$$
\operatorname{dist}(x, G(p)) \leq \frac{1}{\sigma} \operatorname{dist}(0, F(x, p))
$$

for all $(x, p) \in B\left(x_{0}, r / 2\right) \times B\left(p_{0}, r\right)$ with $\operatorname{dist}(0, F(x, p))<\mu$.

Proof. Fix any $\mu \in(0, \min \{r, r \sigma / 2\})$ and any $(x, p) \in$ $B\left(x_{0}, r / 2\right) \times B\left(p_{0}, r\right)$ with $\operatorname{dist}(0, F(x, p))<\mu$. If $\operatorname{dist}(0$, $F(x, p))=0$, then $0 \in F(x, p)$ and hence $x \in G(p)$. Therefore, both sides of (13) are equal to 0 and (13) holds. Hence, we can assume that $\operatorname{dist}(0, F(x, p))=\alpha$, where $\alpha \in(0, \mu)$. It remains to show that

$$
\operatorname{dist}(x, G(p)) \leq \frac{\alpha}{\sigma} .
$$

Since $0<\alpha<\mu<r \sigma / 2$, we obtain that $2 \alpha / r<\sigma$. For each $\varepsilon \in(0, r-\mu)$ with $2(\alpha+\varepsilon) / r<\sigma$, by the definition of the distance function, there exists $\bar{y} \in F_{p}(x)$ such that $\|\bar{y}\|<\alpha+$ $\varepsilon<\mu+\varepsilon<r$. Define the function $f_{p}: X \times Y \rightarrow \overline{\mathbb{R}}$ by

$$
f_{p}\left(x^{\prime}, y^{\prime}\right):=\left\|y^{\prime}\right\|+\delta_{\operatorname{gph}_{p}}\left(x^{\prime}, y^{\prime}\right), \quad \forall\left(x^{\prime}, y^{\prime}\right) \in X \times Y .
$$

We claim that $f_{p}$ is l.s.c. on $X \times Y$ due to condition (i). Fix any $t \in(2(\alpha+\varepsilon) / r, \sigma)$. Put $\beta:=f_{p}(x, \bar{y})=\|\bar{y}\|$. We see that

$$
f_{p}(x, \bar{y})=t \cdot \frac{\beta}{t} \text {. }
$$

Clearly,

$$
f_{p}(x, \bar{y}) \leq \inf _{\left(x^{\prime}, y^{\prime}\right) \in X \times Y} f_{p}\left(x^{\prime}, y^{\prime}\right)+t \cdot \frac{\beta}{t} .
$$

Applying the Ekeland variational principle via the new norm $\left\|\left(x^{\prime}, y^{\prime}\right)\right\|_{\eta}:=\left\|x^{\prime}\right\|+\eta\left\|y^{\prime}\right\|$ in the product space $X \times Y$ for some $0<\eta<1 / \sigma$ allows us to find $(\widehat{x}, \widehat{y}) \in X \times Y$ satisfying

$$
\begin{array}{r}
f_{p}(\widehat{x}, \hat{y}) \leq f_{p}(x, \bar{y}), \quad\|(\widehat{x}, \widehat{y})-(x, \bar{y})\|_{\eta} \leq \frac{\beta}{t}, \\
f_{p}(\widehat{x}, \widehat{y}) \leq f_{p}\left(x^{\prime}, y^{\prime}\right)+t\left\|\left(x^{\prime}, y^{\prime}\right)-(\widehat{x}, \widehat{y})\right\|_{\eta}, \\
\forall\left(x^{\prime}, y^{\prime}\right) \in X \times Y .
\end{array}
$$

This implies that $(\widehat{x}, \hat{y}) \in \operatorname{gph} F_{p}$,

$$
\begin{aligned}
\|\hat{y}\| \leq\|\bar{y}\|, \quad\|\hat{x}-x\|+\eta\|\hat{y}-\bar{y}\| \leq \frac{\beta}{t}, \\
\|\hat{y}\| \leq\left\|y^{\prime}\right\|+t\left(\left\|x^{\prime}-\hat{x}\right\|+\eta\left\|y^{\prime}-\hat{y}\right\|\right) \\
\quad+\delta_{\operatorname{gph}_{p}}\left(x^{\prime}, y^{\prime}\right), \quad \forall\left(x^{\prime}, y^{\prime}\right) \in X \times Y .
\end{aligned}
$$

Furthermore,

$$
\begin{gathered}
\left\|\widehat{x}-x_{0}\right\| \leq\|\hat{x}-x\|+\left\|x-x_{0}\right\| \\
\leq \frac{\beta}{t}+\frac{r}{2}<\frac{\alpha+\varepsilon}{t}+\frac{r}{2}<\frac{r}{2}+\frac{r}{2}=r, \\
\|\hat{y}\| \leq\|\bar{y}\|=\beta<r .
\end{gathered}
$$

That is,

$$
\hat{x} \in \operatorname{int} B\left(x_{0}, r\right) \subset B\left(x_{0}, r\right), \quad \hat{y} \in \operatorname{int} B(0, r) \subset B(0, r) .
$$

We now show that $0 \in F_{p}(\widehat{x})$. Assume to the contrary that $0 \notin F_{p}(\widehat{x})$ and then $\hat{y} \neq 0$. Define the function $\varphi: X \times Y \rightarrow \overline{\mathbb{R}}$ by

$$
\begin{array}{r}
\varphi\left(x^{\prime}, y^{\prime}\right):=\left\|y^{\prime}\right\|+t\left(\left\|x^{\prime}-\widehat{x}\right\|+\eta\left\|y^{\prime}-\widehat{y}\right\|\right), \\
\forall\left(x^{\prime}, y^{\prime}\right) \in X \times Y .
\end{array}
$$

It follows from $(20)$ that $(\hat{x}, \hat{y})$ is a minimum of the function $\varphi+\delta_{\operatorname{gph}_{p}}$ on $X \times Y$. Noting that $\hat{y} \neq 0$, it follows from Lemma 1 that

$$
\begin{aligned}
(0,0) & \in \partial_{c} \varphi(\hat{x}, \hat{y})+\partial_{c} \delta_{g p h F_{p}}(\hat{x}, \hat{y}) \\
& =\{0\} \times J(\hat{y})+t\left(B_{X^{*}} \times \eta B_{Y^{*}}\right)+N_{c}\left((\widehat{x}, \hat{y}) ; g p h F_{p}\right) .
\end{aligned}
$$

This implies that there exist $y_{1}^{*} \in J(\widehat{y})$ and $\left(x_{2}^{*}, y_{2}^{*}\right) \in$ $N_{c}\left((\widehat{x}, \widehat{y}) ; g p h F_{p}\right)$ such that

$$
\left\|x_{2}^{*}\right\| \leq t, \quad\left\|y_{1}^{*}+y_{2}^{*}\right\| \leq t \eta .
$$

It follows that

$$
\left\|y_{2}^{*}\right\| \geq 1-t \eta>0, \quad\left\|y_{2}^{*}\right\| \leq 1+\left\|y_{1}^{*}+y_{2}^{*}\right\| \leq 1+t \eta .
$$


Let $x^{*}:=x_{2}^{*} /\left\|y_{2}^{*}\right\|$ and $y^{*}:=-\left(y_{2}^{*} /\left\|y_{2}^{*}\right\|\right)$. Then $\left(x^{*},-y^{*}\right) \epsilon$ $N_{c}\left((\widehat{x}, \hat{y}) ; \operatorname{gph} F_{p}\right)$, and hence $x^{*} \in D_{c}^{*} F_{p}(\widehat{x}, \hat{y})\left(y^{*}\right)$. We observe that $\left\|y^{*}\right\|=1$ and

$$
\left\|x^{*}\right\|=\frac{\left\|x_{2}^{*}\right\|}{\left\|y_{2}^{*}\right\|} \leq t(1-t \eta)^{-1} .
$$

Since $y_{1}^{*} \in J(\hat{y})$, we have that $\left\|y_{1}^{*}\right\|=1$ and $\left\langle y_{1}^{*}, \hat{y}\right\rangle=\|\hat{y}\|$. Furthermore,

$$
\begin{aligned}
\left\|y_{2}^{*}\right\| \cdot\|\hat{y}\|-\left\|y_{2}^{*}\right\| \cdot\left\langle y^{*}, \hat{y}\right\rangle \\
\quad=\left(\left\|y_{2}^{*}\right\|-1\right)\|\hat{y}\|+\|\hat{y}\|+\left\langle y_{2}^{*}, \hat{y}\right\rangle \\
\quad \leq t \eta r+\left\langle y_{1}^{*}, \hat{y}\right\rangle+\left\langle y_{2}^{*}, \hat{y}\right\rangle \\
\quad=t \eta r+\left\langle y_{1}^{*}+y_{2}^{*}, \hat{y}\right\rangle \\
\quad \leq t \eta r+\left\|y_{1}^{*}+y_{2}^{*}\right\| \cdot\|\hat{y}\| \leq 2 t \eta r .
\end{aligned}
$$

Dividing both sides of the above inequality by $\left\|y_{2}^{*}\right\|$ gives us that

$$
\|\hat{y}\|-\left\langle y^{*}, \hat{y}\right\rangle \leq 2 t \eta r \cdot \frac{1}{\left\|y_{2}^{*}\right\|} \leq 2 t \eta r(1-t \eta)^{-1} .
$$

For any $y^{\prime} \in F_{p}(\widehat{x})$, by (20), we have that

$$
\|\hat{y}\| \leq\left\|y^{\prime}\right\|+t \eta\left\|y^{\prime}-\hat{y}\right\| \leq(1+t \eta)\left\|y^{\prime}\right\|+t \eta\|\hat{y}\| .
$$

This implies that

$$
\|\hat{y}\| \leq \frac{1+t \eta}{1-t \eta}\left\|y^{\prime}\right\|, \quad \forall y^{\prime} \in F_{p}(\widehat{x}) .
$$

Hence,

$$
\begin{aligned}
\|\hat{y}\| & \leq \frac{1+t \eta}{1-t \eta} \operatorname{dist}\left(0, F_{p}(\widehat{x})\right) \\
& =\operatorname{dist}\left(0, F_{p}(\widehat{x})\right)+\frac{2 t \eta}{1-t \eta} \operatorname{dist}\left(0, F_{p}(\widehat{x})\right) .
\end{aligned}
$$

Fix any $\delta>0$; it follows from (27), (29), and (32) that

$$
\left\|x^{*}\right\|<t+\delta, \quad y^{*} \in J_{\delta}(\widehat{y}), \quad \hat{y} \in \Pi_{\delta}\left(0 ; F_{p}(\widehat{x})\right)
$$

when $\eta>0$ are chosen sufficiently small. Taking $\delta \downarrow 0$ in the above gives us that $\left\|x^{*}\right\| \leq t<\sigma$, which is contrary to condition (ii). Therefore, we have shown that $0 \in F_{p}(\widehat{x})$; that is, $\hat{x} \in G(p)$. It follows that

$$
\operatorname{dist}(x, G(p)) \leq\|x-\widehat{x}\| \leq \frac{\beta}{t}<\frac{\alpha+\varepsilon}{t} .
$$

Letting $t \rightarrow \sigma$, we obtain that

$$
\operatorname{dist}(x, G(p)) \leq \frac{\alpha+\varepsilon}{\sigma} .
$$

Letting $\varepsilon \rightarrow 0$, we obtain that

$$
\operatorname{dist}(x, G(p)) \leq \frac{\alpha}{\sigma}=\frac{1}{\sigma} \operatorname{dist}(0, F(x, p)) .
$$

Remark 4. We obtain the same result with Huy et al. [15, Theorem 3.1] under much weaker coderivative condition. Noting that the proof of Theorem 3 is much simpler than that of [15, Theorem 3.1], similar results presented in [9, Theorem 3.5], [10, Proposition 3.6], [14, Corollary 3.3], and [8, Theorem 3.1] all require the assumption of the inner semicompactness of the metric projection mapping. However, Theorem 3 does not require this assumption. Moreover, we can see from the proof of Theorem 3 that the conclusion of the theorem is still valid, if the topological space $P$ is replaced by a metric space.

Theorem 5. Suppose that all the assumptions of Theorem 3 are satisfied. Moreover, assume that

(iii) $F$ is l.s.c. at $\left(x_{0}, p_{0}\right)$.

Then $G$ is metrically regular around $\left(x_{0}, p_{0}\right)$ with modulus $1 / \sigma$. In fact, there exists a constant $\rho>0$ such that

$$
\operatorname{dist}(x, G(p)) \leq \frac{1}{\sigma} \operatorname{dist}(0, F(x, p))
$$

for all $(x, p) \in B\left(x_{0}, \rho\right) \times B\left(p_{0}, \rho\right)$.

Proof. By Theorem 3, for any $\mu \in(0, \min \{r, r \sigma / 2\})$, we have

$$
\operatorname{dist}(x, G(p)) \leq \frac{1}{\sigma} \operatorname{dist}(0, F(x, p))
$$

for all $(x, p) \in B\left(x_{0}, r / 2\right) \times B\left(p_{0}, r\right)$ with $\operatorname{dist}(0, F(x, p))<$ $\mu$. Clearly, $0 \in F\left(x_{0}, p_{0}\right) \cap$ int $B_{\mu}(0)$. By condition (iii), there exists a constant $\rho_{1}>0$ such that

$$
F(x, p) \cap \text { int } B_{\mu}(0) \neq \emptyset, \quad \forall(x, p) \in B\left(x_{0}, \rho_{1}\right) \times B\left(p_{0}, \rho_{1}\right) \text {. }
$$

Hence,

$$
\operatorname{dist}(0, F(x, p))<\mu, \quad \forall(x, p) \in B\left(x_{0}, \rho_{1}\right) \times B\left(p_{0}, \rho_{1}\right) .
$$

Choose a number $\rho \in\left(0, \min \left\{\rho_{1}, r / 2\right\}\right)$. Then $\rho$ satisfies the conclusion of Theorem 5. Indeed, for any $(x, p) \epsilon$ $B\left(x_{0}, \rho\right) \times B\left(p_{0}, \rho\right)$, we have $(x, p) \in B\left(x_{0}, \rho_{1}\right) \times B\left(p_{0}, \rho_{1}\right)$, and it follows from (40) that $\operatorname{dist}(0, F(x, p))<\mu$. Moreover, $(x, p) \in$ $B\left(x_{0}, r / 2\right) \times B\left(p_{0}, r\right)$. It follows from (38) that $\operatorname{dist}(x, G(p)) \leq$ $(1 / \sigma) \operatorname{dist}(0, F(x, p))$.

Remark 6. Similar results presented in [7, Theorem 3.2] and [14, Corollary 3.6] are established in terms of Mordukhovich normal coderivative in Asplund spaces. Moreover, [7, Theorem 3.2] and [14, Corollary 3.6] all require the assumption of the inner semicompactness of the metric projection mapping. However, Theorem 5 does not require this assumption.

Theorem 7. Suppose that all the assumptions of Theorem 3 are satisfied. Moreover, assume that $P$ is a subset of a normed space and

(iii) there exists a constant $l>0$ such that

$$
\begin{array}{r}
F\left(x, p^{\prime}\right) \cap r B_{Y} \subset F(x, p)+l\left\|p^{\prime}-p\right\| B_{Y}, \\
\forall x \in B\left(x_{0}, r\right), \forall p, p^{\prime} \in B\left(p_{0}, r\right) .
\end{array}
$$

Then $G$ is Lipschitz-like around $\left(p_{0}, x_{0}\right)$ with modulus $l / \sigma$. 
Proof. Choose a number $\mu \in(0, \min \{r, r \sigma / 2\})$. We can assert from Theorem 3 that

$$
\operatorname{dist}(x, G(p)) \leq \frac{1}{\sigma} \operatorname{dist}(0, F(x, p))
$$

for all $(x, p) \in B\left(x_{0}, r / 2\right) \times B\left(p_{0}, r\right)$ with $\operatorname{dist}(0, F(x, p))<\mu$. Choose a number $\rho \in(0, r / 2)$ with $2 l \rho<\mu$. We claim that

$$
\begin{array}{r}
G\left(p^{\prime}\right) \cap B\left(x_{0}, \rho\right) \subset G(p)+\frac{l}{\sigma}\left\|p^{\prime}-p\right\| B_{X}, \\
\forall p^{\prime}, p \in B\left(p_{0}, \rho\right) .
\end{array}
$$

Indeed, fix any $p^{\prime}, p \in B\left(p_{0}, \rho\right)$ and any $x \in G\left(p^{\prime}\right) \cap B\left(x_{0}, \rho\right)$. Then we have $0 \in F\left(x, p^{\prime}\right)$, and it follows from condition (iii) that $0 \in F(x, p)+l\left\|p^{\prime}-p\right\| B_{Y}$. Hence,

$$
\begin{aligned}
\operatorname{dist}(0, F(x, p)) & \leq l\left\|p^{\prime}-p\right\| \leq l\left(\left\|p^{\prime}-p_{0}\right\|+\left\|p_{0}-p\right\|\right) \\
& \leq 2 l \rho<\mu .
\end{aligned}
$$

By (42) and (44), we obtain that

$$
\operatorname{dist}(x, G(p)) \leq \frac{1}{\sigma} \operatorname{dist}(0, F(x, p)) \leq \frac{l}{\sigma}\left\|p^{\prime}-p\right\| .
$$

Therefore,

$$
x \in G(p)+\frac{l}{\sigma}\left\|p^{\prime}-p\right\| B_{X} .
$$

It follows that (43) holds. Therefore, $G$ is Lipschitz-like around $\left(p_{0}, x_{0}\right)$ with modulus $l / \sigma$.

Remark 8. We obtain the same result with Huy et al. [15, Theorem 3.2] under much weaker coderivative condition. Similar results presented in [9, Theorem 3.5], [10, Corollary 3.9], [7, Theorem 3.3], and [14, Corollary 3.10] all require the assumption of the inner semicompactness of the metric projection mapping. However, Theorem 7 does not require this assumption. Similar result presented in [12, Theorem 3.1] does not require the assumption of the inner semicompactness of the metric projection mapping, but it is established in terms of Fréchet coderivative in Asplund spaces.

Theorem 9. Suppose that all the assumptions of Theorem 3 are satisfied. Moreover, assume that

(iii) for any $(x, p) \in B\left(x_{0}, r\right) \times B\left(p_{0}, r\right)$, the multifunction $F(x, \cdot)$ is l.s.c. at $p$.

Then there exists a constant $s \in(0, r)$ such that the multifunction $\widetilde{G}: P \rightrightarrows X$ defined by

$$
\widetilde{G}(p):=G(p) \cap \operatorname{int} B\left(x_{0}, r\right)
$$

is nonempty and l.s.c. on $B\left(p_{0}, s\right)$.

Proof. Since $0 \in F\left(x_{0}, p_{0}\right)$, by condition (iii), there exists a constant $\rho>0$ such that

$$
F\left(x_{0}, p\right) \cap \operatorname{int} B(0, \min \{r, r \sigma\}) \neq \emptyset, \quad \forall p \in B\left(p_{0}, \rho\right) .
$$

Hence,

$$
\operatorname{dist}\left(0, F\left(x_{0}, p\right)\right)<\min \{r, r \sigma\}, \quad \forall p \in B\left(p_{0}, \rho\right) .
$$

Choose a number $s \in(0, \min \{r, \rho\})$. We show that $s$ satisfies the conclusion of Theorem 9 .

(a) Fix any $p \in B\left(p_{0}, s\right)$. We prove that $\widetilde{G}(p)$ is nonempty. Define the function $f_{p}: X \times Y \rightarrow \overline{\mathbb{R}}$ by

$$
f_{p}(x, y):=\|y\|+\delta_{\operatorname{gph} F_{p}}(x, y), \quad \forall(x, y) \in X \times Y .
$$

We claim that $f_{p}$ is l.s.c. on $X \times Y$ due to condition (i). If $f_{p}\left(x_{0}, 0\right)=0$, then $0 \in F_{p}\left(x_{0}\right)$, and hence $x_{0} \in G(p)$. It follows that $x_{0} \in G(p) \cap \operatorname{int} B\left(x_{0}, r\right)$. That is, $\widetilde{G}(p) \neq \emptyset$. If $f_{p}\left(x_{0}, 0\right) \neq 0$, then $0 \notin F_{p}\left(x_{0}\right)$, and hence $\operatorname{dist}\left(0, F\left(x_{0}, p\right)\right)>0$. We may assume that $\alpha:=\operatorname{dist}\left(0, F\left(x_{0}, p\right)\right)$, where $0<\alpha<$ $\min \{r, r \sigma\} \leq r$.

For each $\varepsilon \in(0, r-\alpha)$ with $(\alpha+\varepsilon) / r<\sigma$, by the definition of the distance function, there exists $\bar{y} \in F_{p}\left(x_{0}\right)$ such that $\|\bar{y}\|<\alpha+\varepsilon<r$. Let $\beta:=f_{p}\left(x_{0}, \bar{y}\right)=\|\bar{y}\|$. Fix any $t \epsilon$ $((\alpha+\varepsilon) / r, \sigma)$. We see that

$$
f_{p}\left(x_{0}, \bar{y}\right)=t \cdot \frac{\beta}{t} .
$$

Clearly,

$$
f_{p}\left(x_{0}, \bar{y}\right) \leq \inf _{(x, y) \in X \times Y} f_{p}(x, y)+t \cdot \frac{\beta}{t} .
$$

Applying the Ekeland variational principle via the new norm $\|(x, y)\|_{\eta}:=\|x\|+\eta\|y\|$ in the product space $X \times Y$ for some $0<\eta<1 / \sigma$ allows us to find $(\widehat{x}, \widehat{y}) \in X \times Y$ such that

$$
\begin{gathered}
f_{p}(\widehat{x}, \hat{y}) \leq f_{p}\left(x_{0}, \bar{y}\right), \quad\left\|(\widehat{x}, \hat{y})-\left(x_{0}, \bar{y}\right)\right\|_{\eta} \leq \frac{\beta}{t}, \\
f_{p}(\widehat{x}, \widehat{y}) \leq f_{p}(x, y)+t\|(x, y)-(\widehat{x}, \widehat{y})\|_{\eta}, \\
\forall(x, y) \in X \times Y .
\end{gathered}
$$

This implies that $(\widehat{x}, \hat{y}) \in \operatorname{gph} F_{p}$,

$$
\begin{array}{r}
\|\hat{y}\| \leq\|\bar{y}\|, \quad\left\|\hat{x}-x_{0}\right\|+\eta\|\hat{y}-\bar{y}\| \leq \frac{\beta}{t}, \\
\|\hat{y}\| \leq\|y\|+t(\|x-\hat{x}\|+\eta\|y-\hat{y}\|)+\delta_{\operatorname{gph}_{p}}(x, y), \\
\forall(x, y) \in X \times Y .
\end{array}
$$

Furthermore,

$$
\left\|\widehat{x}-x_{0}\right\| \leq \frac{\beta}{t}<\frac{\alpha+\varepsilon}{t}<r, \quad\|\hat{y}\| \leq\|\bar{y}\|<r .
$$

That is,

$$
\hat{x} \in \operatorname{int} B\left(x_{0}, r\right) \subset B\left(x_{0}, r\right), \quad \hat{y} \in \operatorname{int} B(0, r) \subset B(0, r) .
$$


We now show that $0 \in F_{p}(\widehat{x})$. Assume to the contrary that $0 \notin F_{p}(\widehat{x})$ and then $\widehat{y} \neq 0$. Define the function $\varphi: X \times Y \rightarrow \overline{\mathbb{R}}$ by

$$
\begin{array}{r}
\varphi(x, y):=\|y\|+t(\|x-\hat{x}\|+\eta\|y-\hat{y}\|), \\
\forall(x, y) \in X \times Y .
\end{array}
$$

It follows from $(55)$ that $(\widehat{x}, \widehat{y})$ is a minimum of the function $\varphi+\delta_{\mathrm{gph} F_{p}}$ on $X \times Y$. Arguing as in Theorem 3, we can deduce a contradiction with condition (ii). Therefore, we have shown that $0 \in F_{p}(\widehat{x})$; that is, $\widehat{x} \in G(p)$. It follows from (57) that $\widetilde{G}(p) \neq \emptyset$.

(b) Fix any $p \in B\left(p_{0}, s\right)$. We prove that $\widetilde{G}$ is l.s.c. at $p$. It suffices to show that, for any $x \in \widetilde{G}(p)$ and any $\varepsilon>0$, there exists a constant $t>0$ such that

$$
\widetilde{G}\left(p^{\prime}\right) \cap \operatorname{int} B(x, \varepsilon) \neq \emptyset, \quad \forall p^{\prime} \in B(p, t) .
$$

Since $x \in \widetilde{G}(p)$, we have that $0 \in F(x, p)$ and $x \in \operatorname{int} B\left(x_{0}, r\right)$. Choose a number $\eta \in(0, \varepsilon)$ such that $B(x, \eta) \subset B\left(x_{0}, r\right)$ and $B(p, \eta) \subset B\left(p_{0}, r\right)$. Arguing as above for the pair $(x, p)$ in the place of $\left(x_{0}, p_{0}\right)$, the constant $\eta$ in the place of $r$, and the ball $B(x, \eta), B(0, \eta)$, and $B(p, \eta)$ in the place of $B\left(x_{0}, r\right), B(0, r)$, and $B\left(p_{0}, r\right)$, respectively, we find a constant $t \in(0, \eta)$ such that

$$
G\left(p^{\prime}\right) \cap \operatorname{int} B(x, \eta) \neq \emptyset, \quad \forall p^{\prime} \in B(p, t) .
$$

Since int $B(x, \eta) \subset$ int $B\left(x_{0}, r\right) \cap \operatorname{int} B(x, \varepsilon)$, from (60) we get

$$
G\left(p^{\prime}\right) \cap \operatorname{int} B\left(x_{0}, r\right) \cap \operatorname{int} B(x, \varepsilon) \neq \emptyset, \quad \forall p^{\prime} \in B(p, t) .
$$

That is,

$$
\widetilde{G}\left(p^{\prime}\right) \cap \operatorname{int} B(x, \varepsilon) \neq \emptyset, \quad \forall p^{\prime} \in B(p, t) .
$$

Remark 10. Similar results presented in [7, Theorem 3.1] and [14, Theorem 3.12] are established in terms of Mordukhovich normal coderivative in Asplund spaces. Moreover, [7, Theorem 3.1] and [14, Theorem 3.12] all require the assumption of the inner semicompactness of the metric projection mapping. However, Theorem 9 does not require this assumption.

Corollary 11. Let $X$ and $P$ be Banach spaces, $\Phi: X \rightrightarrows P$ a multifunction, and $\left(x_{0}, p_{0}\right) \in X \times P$ a pair with $p_{0} \in \Phi\left(x_{0}\right)$. Suppose that $\Phi$ is closed and that there exist constants $r>0$ and $\sigma>0$ such that, for any $\delta>0$ and any $(x, p) \in B\left(x_{0}, r\right) \times$ $B\left(p_{0}, r\right)$ with $p \notin \Phi(x)$,

$$
\begin{aligned}
& \sigma \leq \liminf _{\delta \downarrow 0}\left\{\left\|x^{*}\right\|: x^{*} \in D_{c}^{*} \Phi(x, y+p)\left(y^{*}\right),\right. \\
& \left.y \in \Pi_{\delta}(0 ; \Phi(x)-p) \cap B(0, r), y^{*} \in J_{\delta}(y)\right\},
\end{aligned}
$$

where $\Pi_{\delta}(0 ; \Phi(x)-p):=\{y \in \Phi(x)-p \mid\|y\| \leq \operatorname{dist}(0, \Phi(x)-$ $p)+\delta\}$ and $J_{\delta}(y):=\left\{y^{*} \in S_{Y^{*}} \mid\|y\|-\left\langle y^{*}, y\right\rangle \leq \delta\right\}$.

\section{Then one has the following:}

(a) there exists a constant $\rho_{1}>0$ such that, for any $\tau \in$ $\left(0, \rho_{1}\right], B\left(p_{0}, \sigma \tau\right) \subset \Phi\left(B\left(x_{0}, \tau\right)\right) ;$

(b) there exist constants $\mu>0$ and $\rho_{2}>0$ such that

$$
\operatorname{dist}\left(x, \Phi^{-1}(p)\right) \leq \frac{1}{\sigma} \operatorname{dist}(p, \Phi(x))
$$

for all $(x, p) \in B\left(x_{0}, \rho_{2}\right) \times B\left(p_{0}, \rho_{2}\right)$ satisfying $\operatorname{dist}(p, \Phi(x)) \leq \mu$;

(c) $\Phi^{-1}$ is Lipschitz-like around $\left(p_{0}, x_{0}\right)$ with modulus $1 / \sigma$;

(d) there exists a constant $s \in(0, r)$ such that the multifunction $\widetilde{G}: P \rightrightarrows X$ defined by

$$
\widetilde{G}(p):=\Phi^{-1}(p) \cap \operatorname{int} B\left(x_{0}, r\right)
$$

is nonempty and l.s.c. on $B\left(p_{0}, s\right)$.

Proof. Put $Y:=P$. Define $F: X \times P \rightrightarrows Y$ and $G: P \rightrightarrows X$ by

$$
\begin{aligned}
& F(x, p):=\Phi(x)-p, \quad \forall(x, p) \in X \times P, \\
& G(p):=\{x \in X: 0 \in F(x, p)\}, \quad \forall p \in P,
\end{aligned}
$$

respectively. Obviously, $G(p)=\{x \in X: p \in \Phi(x)\}=$ $\Phi^{-1}(p)$. It is easy to see that all the assumptions of Theorem 3 are satisfied. Indeed, $p_{0} \in \Phi\left(x_{0}\right)$ implies that $0 \in F\left(x_{0}, p_{0}\right)$. Denote $F_{p}(\cdot):=F(\cdot, p)$. We observe that $g \mathrm{ph} F_{p}=\operatorname{gph} \Phi-$ $(0, p)$ and $\forall p \in P$. Since $\Phi$ is closed, we have that $F_{p}$ is closed for all $p \in P$. It follows that condition (i) of Theorem 3 is satisfied. Furthermore, we can prove that $T_{c}\left((x, y) ; g \mathrm{ph} F_{p}\right)=$ $T_{c}((x, y+p) ; \operatorname{gph} \Phi)$, and it follows that $N_{c}\left((x, y) ; g p h F_{p}\right)=$ $N_{c}((x, y+p)$; gph $\Phi)$. Hence, $x^{*} \in D_{c}^{*} F_{p}(x, y)\left(y^{*}\right) \Leftrightarrow$ $x^{*} \in D_{c}^{*} \Phi(x, y+p)\left(y^{*}\right)$. Then condition (ii) of Theorem 3 is satisfied.

Fix any $\mu \in(0, \min \{r, r \sigma / 2\})$. By Theorem 3 , we have

$$
\operatorname{dist}\left(x, \Phi^{-1}(p)\right) \leq \frac{1}{\sigma} \operatorname{dist}(p, \Phi(x))
$$

for all $(x, p) \in B\left(x_{0}, r / 2\right) \times B\left(p_{0}, r\right)$ with $\operatorname{dist}(p, \Phi(x))<\mu$. We now prove the conclusions of the corollary.

(a) Choose a number $\rho_{1} \in(0, \mu / \sigma)$. Let $\tau \in\left(0, \rho_{1}\right]$. Take arbitrary $p \in B\left(p_{0}, \sigma \tau\right)$. Clearly, $p \in B\left(p_{0}, \sigma \rho_{1}\right) \subset$ $B\left(p_{0}, \mu\right) \subset B\left(p_{0}, r\right)$. Since $p_{0} \in \Phi\left(x_{0}\right)$, we have that $\operatorname{dist}\left(p, \Phi\left(x_{0}\right)\right) \leq\left\|p-p_{0}\right\|<\mu$. It follows from (67) that

$$
\begin{aligned}
\operatorname{dist}\left(x_{0}, \Phi^{-1}(p)\right) & \leq \frac{1}{\sigma} \operatorname{dist}\left(p, \Phi\left(x_{0}\right)\right) \\
& \leq \frac{\operatorname{dist}\left(p, \Phi\left(x_{0}\right)\right)-\operatorname{dist}\left(p_{0}, \Phi\left(x_{0}\right)\right)}{\sigma} \\
& \leq \frac{\left\|p-p_{0}\right\|}{\sigma} \leq \tau .
\end{aligned}
$$

Hence, $B\left(p_{0}, \sigma \tau\right) \subset \Phi\left(B\left(x_{0}, \tau\right)\right)$. 
(b) Take any $\rho_{2} \in(0,(r / 2)]$. We can get the conclusion immediately from (67).

(c) Clearly, $\Phi(x)-p^{\prime} \subset \Phi(x)-p+p-p^{\prime} \subset \Phi(x)-$ $p+\left\|p^{\prime}-p\right\| B_{Y}$. We can verify that condition (iii) of Theorem 7 holds for $F$ with modulus $l=1$. The conclusion follows immediately from Theorem 7 .

(d) Clearly, for any $(x, p) \in B\left(x_{0}, r\right) \times B\left(p_{0}, r\right)$, the multifunction $F(x, \cdot)$ is l.s.c. at $p$. The conclusion follows immediately from Theorem 9 .

Remark 12. We obtain the same result with Huy et al. [15, Corollary 3.1] under much weaker coderivative condition. Similar results presented in [10, Corollary 3.10] are established in terms of Mordukhovich normal coderivative in Asplund spaces. Moreover, [10, Corollary 3.10] requires the assumption of the inner semicompactness of the metric projection mapping. However, Corollary 11 does not require this assumption.

\section{Conflict of Interests}

The authors declare that there is no conflict of interests regarding the publication of this paper.

\section{Acknowledgments}

This work was supported by the National Natural Science Foundation of China (nos. 11226228, 71372113, and 11301254), the Science and Technology Program Project of Henan Province of China (no. 122300410256), and the Natural Science Foundation of Henan Education Department of China (no. 2011B110025).

\section{References}

[1] S. M. Robinson, "Stability theory for systems of inequalities, I. Linear systems," SIAM Journal on Numerical Analysis, vol. 12, no. 5, pp. 754-769, 1975.

[2] S. M. Robinson, "Stability theory for systems of inequalities, II. Differentiable nonlinear systems," SIAM Journal on Numerical Analysis, vol. 13, no. 4, pp. 497-513, 1976.

[3] S. M. Robinson, "Generalized equations and their solutions, I. Basic theory," Mathematical Programming Studies, vol. 10, pp. 128-141, 1979.

[4] S. M. Robinson, "Generalized equations and their solutions, II. Applications to nonlinear programming," Mathematical Programming Studies, vol. 19, pp. 200-221, 1982.

[5] Y. S. Ledyaev and Q. J. Zhu, "Implicit multifunction theorems," Set-Valued Analysis, vol. 7, no. 3, pp. 209-238, 1999.

[6] H. V. Ngai and M. Théra, "Error bounds and implicit multifunction theorem in smooth Banach spaces and applications to optimization," Set-Valued Analysis, vol. 12, no. 1-2, pp. 195-223, 2004.

[7] G. M. Lee, N. N. Tam, and N. D. Yen, "Normal coderivative for multifunctions and implicit function theorems," Journal of Mathematical Analysis and Applications, vol. 338, no. 1, pp. 1122, 2008.
[8] N. D. Yen and J.-C. Yao, "Point-based sufficient conditions for metric regularity of implicit multifunctions," Nonlinear Analysis: Theory, Methods and Applications, vol. 70, no. 7, pp. 2806-2815, 2009.

[9] N. Q. Huy and J.-C. Yao, "Stability of implicit multifunctions in Asplund spaces," Taiwanese Journal of Mathematics, vol. 13, no. 1, pp. 47-65, 2009.

[10] N. Q. Huy and J.-C. Yao, "Metric regularity of parametric generalized inequality systems," Taiwanese Journal of Mathematics, vol. 14, no. 5, pp. 2107-2123, 2010.

[11] N. H. Chieu, J.-C. Yao, and N. D. Yen, "Relationships between Robinson metric regularity and Lipschitz-like behavior of implicit multifunctions," Nonlinear Analysis: Theory, Methods and Applications, vol. 72, no. 9-10, pp. 3594-3601, 2010.

[12] T. D. Chuong, "Lipschitz-like property of an implicit multifunction and its applications," Nonlinear Analysis: Theory, Methods and Applications, vol. 74, no. 17, pp. 6256-6264, 2011.

[13] T. T. A. Nghia, "A note on implicit multifunction theorems," Optimization Letters, vol. 8, no. 1, pp. 329-341, 2014.

[14] M. G. Yang and N. J. Huang, "Random implicit function theorems in Asplund spaces with applications," Journal of Nonlinear and Convex Analysis, vol. 14, no. 3, pp. 497-517, 2013.

[15] N. Q. Huy, D. S. Kim, and K. V. Ninh, "Stability of Implicit Multifunctions in Banach Spaces," Journal of Optimization Theory and Applications, vol. 155, pp. 558-571, 2012.

[16] B. S. Mordukhovich, Variational Analysis and Generalized Differentiation vol I: Basic Theory, Springer, Berlin, Germany, 2006.

[17] B. S. Mordukhovich, Variational Analysis and Generalized Differentiation vol II: Applications, Springer, Berlin, Germany, 2006.

[18] F. H. Clarke, Optimization and Nonsmooth Analysis, Wiley, New York, NY, USA, 1983. 


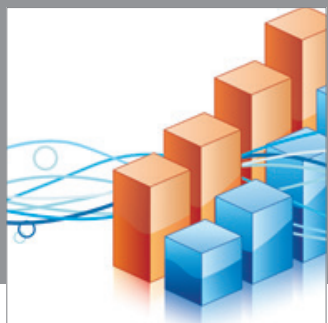

Advances in

Operations Research

mansans

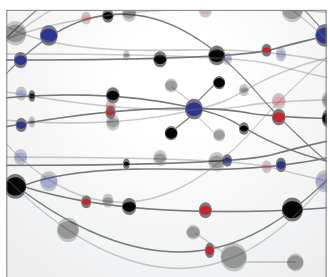

The Scientific World Journal
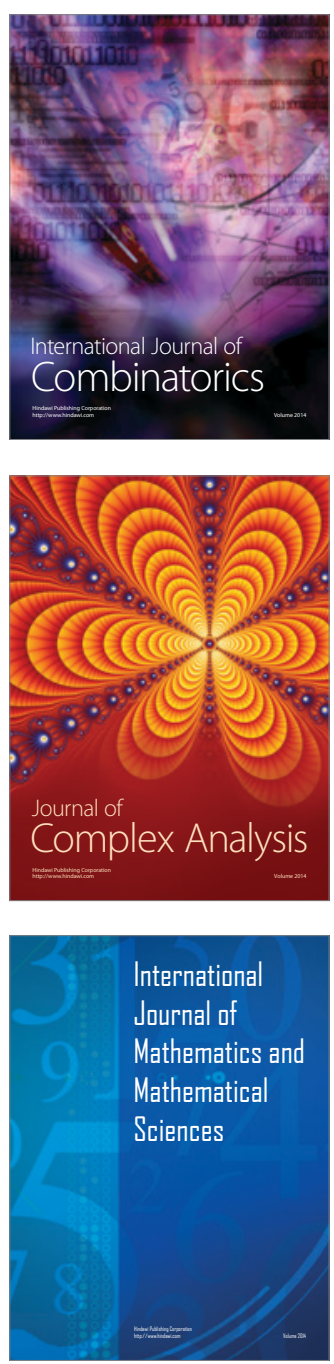
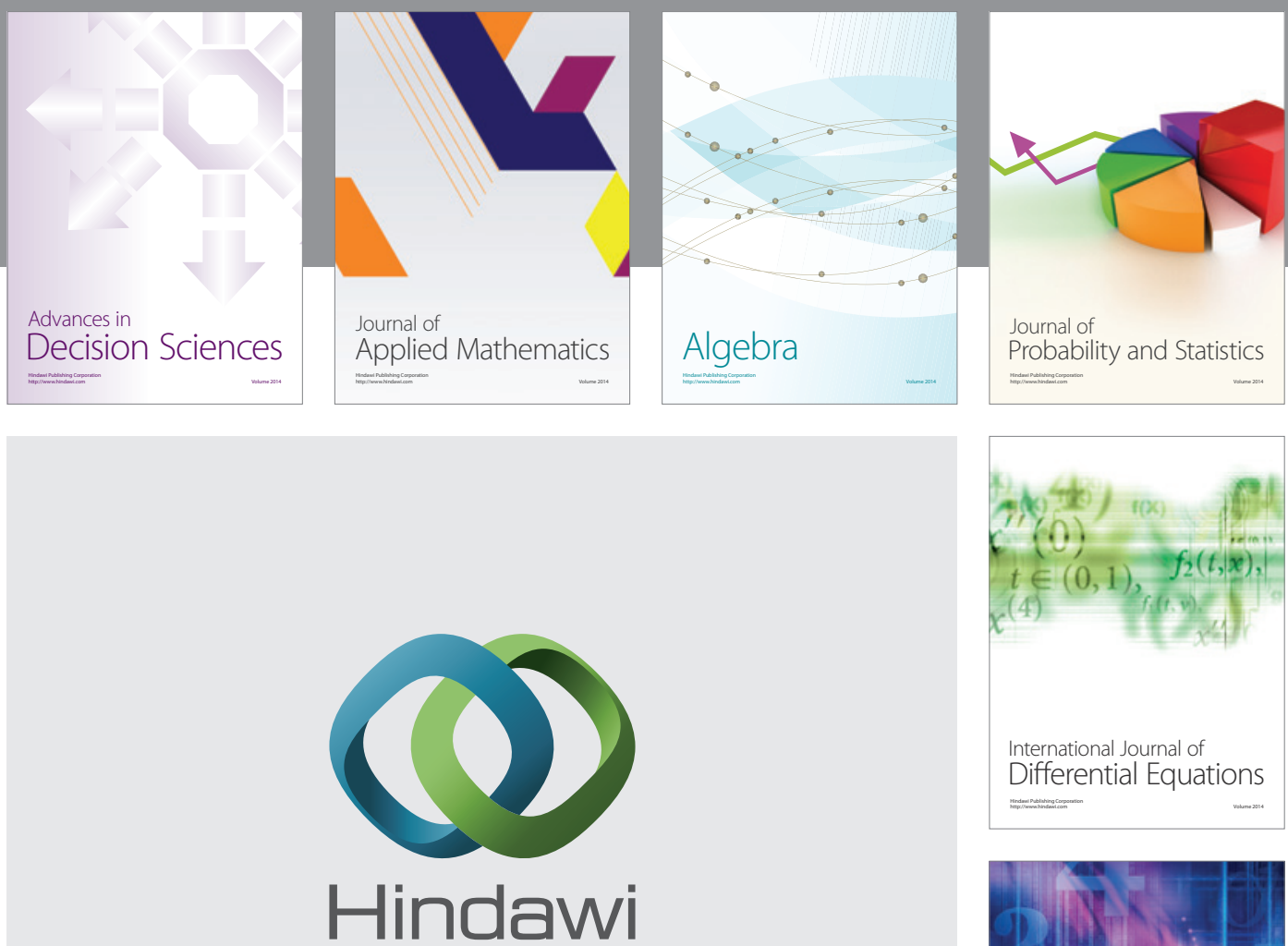

Submit your manuscripts at http://www.hindawi.com
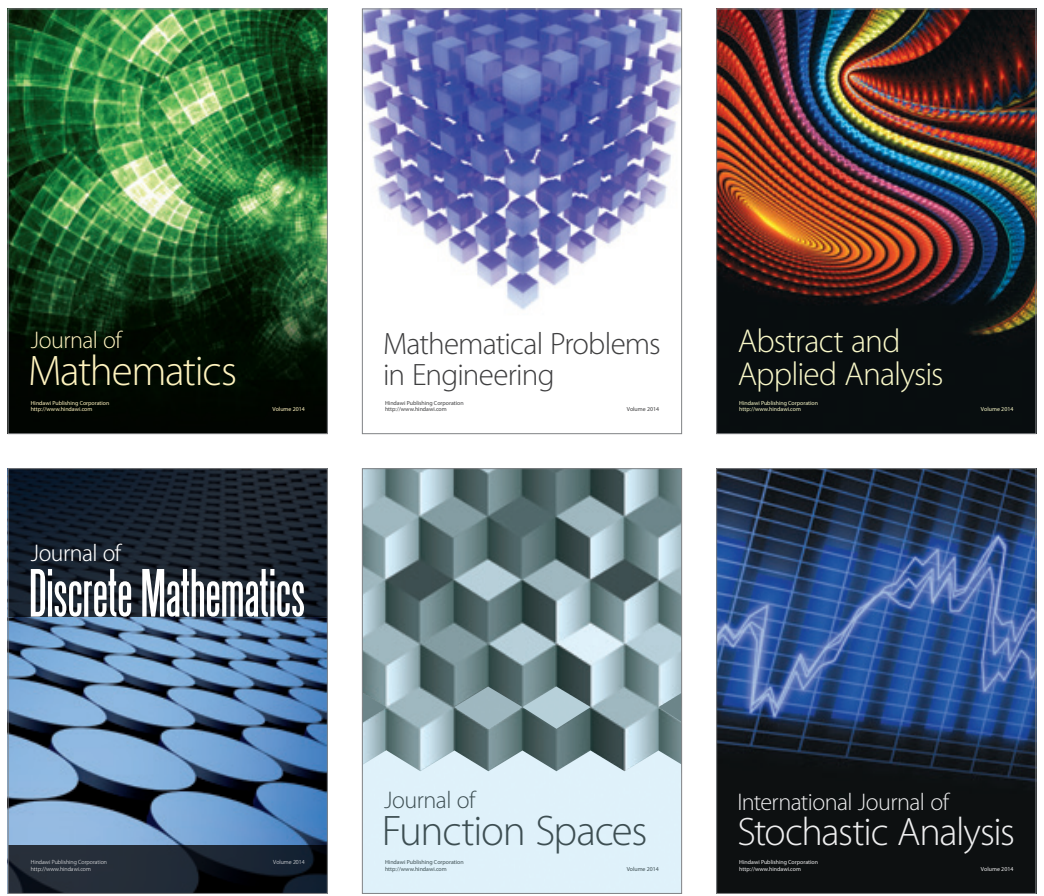

Journal of

Function Spaces

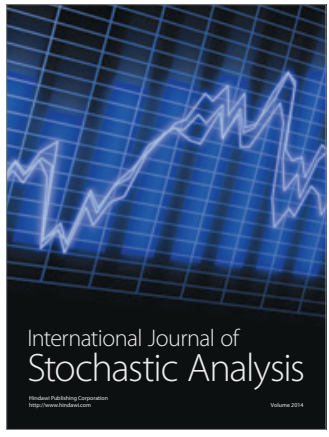

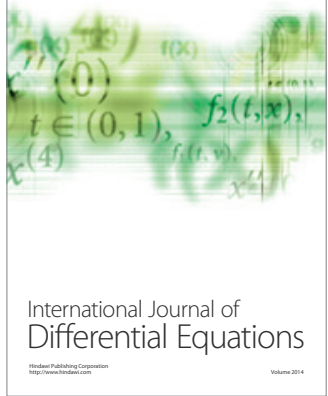
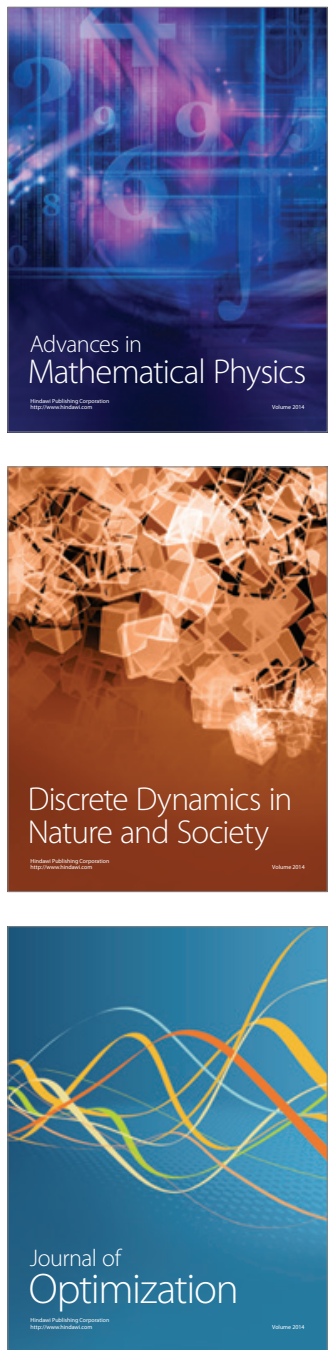